\title{
Kommentar I zum Fall: „Heimlicher Transfer von HIV-Medikamenten nach Afrika“"
}

\author{
Tanja Krones · Christine Angelika Rüegg • \\ Huldrych Fritz Günthard • Annette Rid • Verina Wild
}

Dieser Fall berührt zutiefst, indem er ein für Menschen in Westeuropa beinahe unvorstellbares Lebensschicksal schildert.

Es geht um die bestmögliche Behandlung einer Patientin, die nicht - wie anfangs vermutet - bezüglich ihrer Langzeit-HIV-Therapie ,,incompliant“ ist, sondern einen Teil der für sie verschriebenen antiretroviralen Medikamente zu ihrem Bruder nach Afrika schickt, der an Aids erkrankt ist. Nach Aussagen der Patientin geht es dem Bruder dadurch besser. Die behandelnden Ärzte erläutern der Patientin die Konsequenzen ihres Handelns. Wenn sowohl die Patientin als auch der Bruder zu wenig der HIV-Medikamente erhalten, könnten sich bei beiden Virusresistenzen entwickeln, die ein komplexeres Therapieregime erforderlich machen. Wenn die Ärzte dem Wunsch der Patientin folgen und die doppelte Dosis an Medikamenten verschreiben, würde dies die Krankenkasse zudem über kurz oder lang bemerken. Wie es scheint, wird die Patientin jedoch trotzdem weiterhin Medikamente zu ihrem Bruder schicken - und zwar unabhängig davon, ob sie die für sie notwendige einfache Medikamentendosis oder die doppelte Dosis erhält. Verschreiben die Ärzte die doppelte Dosis, wird die Patientin vermutlich eine für sie ausreichende Behandlung erhalten und zudem wird sich vermutlich der Gesundheitszustand des Bruders der Patientin weiterhin verbessern. Die Krankenkasse würde dann aber die Medikamente für zwei statt für einen Patienten bezahlen, wobei der eine Patient nicht Mitglied der Krankenkasse ist.

Doch auch wenn dies nicht auffiele (was auf die Dauer unwahrscheinlich ist), erscheint diese Lösung problematisch. Es werden Gelder der Solidargemeinschaft für einen Patienten verwendet, der dieser Solidargemeinschaft nicht angehört. Zunächst bestehen also direkte Hilfspflichten nur der im Lande versicherten Patientin gegenüber. Man könnte argumentieren, dass die Patientin eigenverantwortlich entscheiden kann und die behandelnden Ärzte für einen entstehenden Schaden nicht verantwortlich sind, da die Patientin aufgeklärt wurde

PD Dr. med. Dipl. Soz T. Krones $(\bowtie) \cdot$ Dr. med. C. A. Rüegg ·

Prof. Dr. med. H. F. Günthard · Dr. med. A. Rid · Dr. med. V. Wild

Universitätsspital Zürich, DERM C 18,

Gloriastrasse 31, 8091 Zürich, Schweiz

E-Mail: tanja.krones@usz.ch 
und sie die Problematik verstanden hat. Man kann auf dieser Basis überlegen, die Medikamente nur für einige wenige Tage mitzugeben und/oder eine Abgabe der Medikation unter Aufsicht zu veranlassen. Letzteres ist jedoch nur eine hypothetische Möglichkeit, da die Patientin nicht drogenabhängig oder psychisch krank ist, und erscheint zudem kontraproduktiv. Diese Option würde der Fürsorge und dem Wunsch, der Patientin nicht zu schaden, wenig entsprechen und das Vertrauensverhältnis eventuell so belasten, dass sich die Patientin der Behandlung entzieht.

Doch es existiert darüber hinaus eine weitere Ebene, die wir auch aus individueller Perspektive als moralisch problematisch empfinden können: Es bestehen globale Ungerechtigkeiten in Bezug auf die Verteilung von Gütern und den Zugang zu Gesundheitsleistungen. Dadurch, dass die Ärztinnen und Ärzte nun von dem Bruder Kenntnis haben, erhält die Ungerechtigkeit ein Gesicht und sie könnten sich durch die „rule of rescue“ verpflichtet fühlen zu helfen (nach AR Jonsen). Diese als starke menschliche Neigung bezeichnete ,rule of rescue" besagt, dass wir uns identifizierbaren Menschen gegenüber stärker verpflichtet fühlen als den ,nameless faces“, daher dem Bruder der Patientin eventuell mehr als den vielen anderen Menschen, die ebenfalls weltweit an Aids sterben.

In diesem Fall wird uns die global bestehende Ungerechtigkeit jedoch nicht nur sehr direkt offenbar, sie ist auch mit den direkten Hilfspflichten für die Patientin verknüpft. Wir ahnen aufgrund der sozialen und Beziehungssituation, dass die Patientin auf Kosten ihrer eigenen Gesundheit weiter Medikamente zu ihrem Bruder schicken wird. Man mag aus den Angaben des Falls zudem spekulieren, dass die traumatische Lebensgeschichte der Patientin zu einem Selbstbild geführt hat, nicht viel wert zu sein und sich aufopfern zu müssen. Weiter mag die Tatsache, dass die Patientin früher selbst Aids-Kranke behandelt hat, die in Afrika häufig schnell sterben, dazu führen, dass sie weniger Glauben an die Effektivität der Medikamente hat, als diese real für sie selbst haben können. Dies lässt an verschiedene Interventionsmöglichkeiten (Patientenbildung, Aufarbeitung der Traumata etc.) auf der individuellen Ebene denken, was möglicherweise die Situation verbessert. Das Dilemma, dass die Patientin ihrem Bruder auf Kosten ihrer eigenen Gesundheit weiter helfen wollen wird, ist damit nicht gelöst.

Darüber hinaus heißt es in der Genfer Deklaration des Weltärztebundes, dass jeder Arzt nicht nur der Gesundheit des Kranken sondern auch der Humanität insgesamt verpflichtet sei. Dieser bislang wenig diskutierte, über direkte Verpflichtungen eigenen Patienten gegenüber hinausgehende Impetus des Genfer Gelöbnisses, der auch Menschen in anderen Ländern einschließt, macht aus indirekten Hilfspflichten gegenüber „,der Menschheit“ ein den Arzt direkt bindendes moralisches Prinzip. Damit berührt dieser Fall den noch jungen Bereich der „Global Ethics“ [3]: Wie viel schulden wir als Individuum oder insgesamt als Gesellschaft denjenigen, die nicht in unserem Land leben, denen wir uns aber als Menschen verbunden fühlen (können)? Das Spektrum der Auffassungen reicht von liberalen Ansichten, dass wir „denen überhaupt nichts schulden“, da wir an der Entstehung ihres Leidens nicht beteiligt sind [2], bis zu Vertretern des „Cosmopolitanism“ (z. B. [1, 4]), die direkte moralische Pflichten jedes Menschen gegenüber jedem anderen Menschen der Welt als gegeben oder zumindest konstruierbar ansehen.

Die Ärztinnen und Ärzte können sich also sowohl im Sinne der direkten Hilfspflichten für die Patientin als auch im Sinne der indirekten Hilfspflichten gegenüber der Menschheit moralisch verpflichtet fühlen, mehr zu tun als lediglich der Patientin ihre Einzeldosis zu verschreiben. Es ist jedoch schwer vertretbar, für die Dosis des Bruders die gesetzlichen Krankenkassen zu belasten. Das Budget der humanitären Hilfe wäre hierfür geeigneter. So könnte dieser Fall Anlass geben, sich für einen Hilfsfonds am Krankenhaus einzusetzen, der soziale Härtefälle innerhalb und sogar außerhalb der eigenen Staatsgrenzen abdeckt. Mit 
derartigen strukturellen Lösungen wäre auch dem Umstand Rechnung getragen, dass nicht nur dem einzelnen Bruder geholfen würde, sondern in vergleichbaren Fällen auch andere davon profitieren könnten.

Das gebotene Ausmaß von Engagement über die eigenen Staatsgrenzen hinaus ist auch aus der Debatte um „Global Ethics“ heraus nicht klar bestimm- oder einforderbar. Ärztinnen und Ärzte, die sich dem kosmopolitischen Impetus des Genfer Gelöbnisses tugendethisch verpflichtet sehen, werden jedoch eher diesen Weg gehen, der in einer Welt zunehmender Globalisierung und globaler Ungerechtigkeit als Vorbild dienen kann.

\section{Literatur}

1. Brock G (2009) Global justice. A cosmopolitan account. Oxford University Press, Oxford

2. Narveson J (2003) We don't owe them a thing! A tough-minded but soft-hearted view of aid to the faraway needy. Monist 86:419-433

3. Pogge T, Horton K (Hrsg) (2008) Global ethics: seminal essays. Paragon House, St Paul

4. Young IM (2006) Responsibility and global justice: a social connection model. Soc Philos Policy 23(1):102-130 